\title{
DECOMPOSITION THEOREMS IN RATIONAL HOMOTOPY THEORY
}

\author{
JOHN OPREA
}

\begin{abstract}
Let $F \rightarrow E \rightarrow B$ be a fibration of simply connected rational spaces with finite rational betti numbers. Denote the connecting homomorphism of the fibration by $\partial_{\#}$ and the Hurewicz map of the fibre $F$ by $h$. Then, it is shown that there is a decomposition $F \simeq \mathcal{F} \times K$ where $K$ is the product of rational Eilenberg-Mac Lane spaces contained in $\Omega B$ maximal with respect to the conditions: $\pi_{*}(K) \cap \operatorname{Ker} \partial_{\#}=0$ and $\partial_{\#}\left(\pi_{*}(K)\right) \cap \operatorname{Ker} h=0$.

This decomposition is obtained using Sullivan's theory of minimal models. Applications are given of the main theorem and a dual result is proved for rational cofibrations.
\end{abstract}

Conventions and notation. Throughout this note we shall assume that all spaces under consideration are of the homotopy type of simply connected, rational CW complexes with finite rational betti numbers. Such spaces, for example, are obtained as the rationalizations of simply connected $\mathrm{CW}$ complexes of finite type. (See $[\mathbf{G M}]$.) For $X$ as above, we shall write (unambiguously) $H^{*}(X), H_{*}(X)$ and $\pi_{*}(X)$ for the rational cohomology algebra, rational homology coalgebra and rational homotopy Whitehead algebra respectively.

1. Introduction. In studying the homogeneous space fibration

$$
G / K \rightarrow B K \rightarrow B G
$$

Cartan $[\mathbf{C}]$ exploited the interplay between the connecting homomorphism in the homotopy sequence of the fibration and the Hurewicz map of $G / K$ to compute $H^{*}(G / K ; \mathbf{R})$. (For a rational homotopy version of Cartan's result, see [HT, $\left.\S 4\right]$.) In his analysis, Cartan developed a differential graded algebra technique which inspired Sullivan's theory of minimal models. What this suggests is that a proper setting for a more general study of the relationship between the connecting homomorphism of a fibration and the Hurewicz map of the fibre may be found in the framework of rational homotopy theory. In this paper we will make some initial attempts to justify this belief. In particular, we use the relationship between the Hurewicz map and the connecting homomorphisms of a fibration and a cofibration to obtain product and wedge decompositions of the fibre and cofibre respectively.

In the period between Cartan's result and the development of minimal model theory, the relationship described above found much application. In particular, the work of Gottlieb (e.g., [G1, G2]) contains many interesting results in this direction. Also, it was at this time that the powerful effect of the connecting homomorphism on the homotopy type of the fibre became better understood. The following result, originally discovered by Haslam $[\mathbf{H}]$ in connection with work on

Received by the editors March 20, 1984 and, in revised form, April 22, 1985.

1980 Mathematics Subject Classification. Primary 55P62, 55Q99. 
evaluation subgroups, exemplifies this effect. (We remind the reader to recall our conventions!)

THEOREM A $[\mathbf{H}, \mathbf{H 1}, \mathbf{F T}]$. If $F \rightarrow E \stackrel{p}{\rightarrow} B$ is a fibration and $\partial_{\#}$ is surjective, then $F$ has the homotopy type of a product of Eilenberg-Mac Lane spaces.

REMARK. (1) We have stated Theorem A in this form because we wish to emphasize the condition on the connecting homomorphism $\partial_{\#}: \pi_{*+1}(B) \rightarrow \pi_{*}(F)$. The exactness of the homotopy sequence of a fibration implies, however, that we could have imposed the equivalent condition that $p_{\#}: \pi_{*}(E) \rightarrow \pi_{*}(B)$ be injective.

(2) Note that the description of $F$ implies that the Hurewicz map $h: \pi_{*}(F) \rightarrow$ $H_{*}(F)$ is injective. Compare this to Theorem 1 below.

The proof of Theorem A is short and provides many of the elements used in later sections.

ProOF OF THEOREM A. The dual Puppe sequence of the fibration provides a map $\partial: \Omega B \rightarrow F$ inducing $\partial_{\#}$ via the identification $\pi_{*+1}(B) \cong \pi_{*}(\Omega B)$. Now, with respect to our conventions, $\Omega B$ has the homotopy type of a product of EilenbergMac Lane spaces. Therefore, we may choose a subproduct $K \subset \Omega B$ such that the restriction of $\partial$ to $K$ induces isomorphisms in homotopy. Hence $F$ has the homotopy type of $K$. Q.E.D.

The role of Eckmann-Hilton duality in topology provides a secondary theme for this paper. There is an interaction between the connecting homomorphism in the homology sequence of a cofibration and the Hurewicz map of the cofibre which dualizes the situation alluded to earlier for the case of a fibration. Before we discuss the effects of these dual relationships, we note that Theorem A (and its proof) may be dualized to yield,

THEOREM B [FT]. If $X \stackrel{f}{\rightarrow} Y \rightarrow C$ is a cofibration and $\partial_{*}$ is injective, then $C$ has the homotopy type of a wedge of spheres.

REMARK. (1) The condition on $\partial_{*}: H_{*+1}(C) \rightarrow H_{*+1}(\Sigma X) \cong H_{*}(X)$ may be replaced by the equivalent condition that $f_{*}: H_{*}(X) \rightarrow H_{*}(Y)$ be surjective.

(2) Note that the Hurewicz map $h: \pi_{*}(C) \rightarrow H_{*}(C)$ is surjective; compare with Theorem 2 below.

(3) $C$ has the homotopy type of the maximal subwedge of $\Sigma X \simeq \bigvee S^{r_{j}}$ such that $\partial_{*}$ is surjective.

We now come to the decomposition results which form the basis of this investigation. Note the heavy dependence on the $\partial_{\#-h}$ and $\partial_{*}-h$ interaction. In order to understand the statements of the theorems, we remind the reader that, for any $X$ subject to our conventions, $\Omega X$ has the type of a product of Eilenberg-Mac Lane spaces and $\Sigma X$ has the type of a wedge of spheres. Also, we denote the image of the Hurewicz map $h: \pi_{*}(X) \rightarrow H_{*}(X)$ by $S(X)$.

THEOREM 1. If $F \rightarrow E \rightarrow B$ is a fibre sequence, then

$$
F \simeq \mathcal{F} \times K
$$

where $K$ is the subproduct of $\Omega B$ maximal with respect to the conditions, $\pi_{*}(K) \cap$ $\operatorname{Ker} \partial_{\#}=0$ and $\partial_{\#}\left(\pi_{*}(K)\right) \cap \operatorname{Ker} h=0$.

THEOREM 2. If $X \rightarrow Y \rightarrow C$ is a cofibre sequence, then

$$
C \simeq C \vee S
$$

where $S$ is the maximal subwedge of $\Sigma X$ such that $\left.\partial_{*}\right|_{S(C)}$ is surjective. 
REMARK. Theorem 1 may be used to translate conditions on $F$ into information about the evaluation subgroups of $F$ (see $[\mathbf{G 1}]$ for a definition). For example, if all cup products in $H^{*}(F)$ vanish and $F$ does not have the homotopy type of an odd-dimensional sphere, then it is clear that $K$ must be trivial. (Compare with Theorem 5-2 of $[\mathbf{G 1}]$.) In turn, because this holds for any fibration with fibre $F$, we see that the evaluation subgroup of $F$ must be contained in the kernel of the Hurewicz map. This result is very much in the spirit of $[\mathbf{G 1}, \mathbf{G 2}]$. As an example of a space satisfying the conditions above (and which is not a wedge of spheres) take $F=\left(S^{n} \vee S^{n}\right) \cup_{\alpha} e^{3 n-2}$, where $\alpha$ represents the Whitehead product $\left[i_{1},\left[i_{1}, i_{2}\right]\right]$ and $i_{1}, i_{2}$ represent the respective inclusions of $S^{n}$ in $S^{n} \vee S^{n}$. We hope to consider these types of applications further, as well as dual results, in a later paper.

As indicated by our earlier remarks, it is our point of view that Theorems 1 and 2 have their most natural formulations and proofs in the framework of rational homotopy theory. An additional advantage of this algebraic setting is that the duality between Theorems 1 and 2 becomes quite obvious. For these reasons the remainder of the paper is cast in the language of minimal models. We begin by briefly reviewing this theory.

2. Minimal models. Recall that $L(V)$ denotes the commutative graded algebra freely generated by the vector space $V$. That is, $L(V)$ is the tensor product of a polynomial algebra on even-dimensional generators and an exterior algebra on odd-dimensional generators. If $M=L(V)$ is provided with a graded derivation $d$ of degree 1 satisfying $d^{2}=0$ and $d\left(M^{+}\right) \subset M^{+} \cdot M^{+}$, then $(M, d)$ is said to be a minimal differential graded algebra (DGA). To any space $X$, there is associated a minimal DGA model $M(X)$ which describes the homotopy type of $X$. (We remind the reader to recall our conventions.) In particular, there is a natural isomorphism

$$
H^{*}(M(X)) \cong H^{*}(X)
$$

Furthermore, the indecomposables of $M(X), Q M(X)=M(X)^{+} / M(X)^{+} \cdot M(X)^{+}$, are dual to the homotopy groups of $X$; that is,

$$
Q^{i}(M(X)) \cong \operatorname{Hom}\left(\pi_{i} X, \mathbf{Q}\right) .
$$

As shorthand, we write $Q X$ for $Q M(X)$. Finally, we note that any map $f: X \rightarrow Y$ induces a DGA map $F: M(Y) \rightarrow M(X)$ which is unique up to DGA-homotopy. For details see [GM, H2 or H3] for example.

The dual approach to rational homotopy theory is via differential graded Lie algebras (DGLA). Let $F[V]$ denote the free graded Lie algebra on the vector space $V$. If $\mathcal{L}=F[V]$ is given a graded derivation $d$ of degree -1 satisfying $d^{2}=0$ and $d(\mathcal{L}) \subset[\mathcal{L}, \mathcal{L}]$, then $(\mathcal{L}, d)$ is called a minimal DGLA. A space $X$ has a minimal DGLA model $\mathcal{L}(X)$ which describes its homotopy type; in particular,

$$
H \mathcal{L}(X) \cong \pi_{*}(\Omega X) \text { and } \quad Q \mathcal{L}(X) \cong s^{-1} \tilde{H}_{*}(X) .
$$

Here $Q \mathcal{L}(X)$ denotes the indecomposables of $\mathcal{L}(X), \mathcal{L}(X) /[\mathcal{L}(X), \mathcal{L}(X)]$. A map $f: X \rightarrow Y$ induces a DGLA map $F: \mathcal{L}(X) \rightarrow \mathcal{L}(Y)$ which is unique up to DGLA homotopy. The DGLA approach was introduced in $[\mathbf{Q}]$ and developed in $[\mathbf{N}$ and BL]. The most complete treatment, however, may be found in the recent book of Tanre $[\mathbf{T}]$. 
Each of the approaches above yields a description of the Hurewicz homomorphism:

$$
h^{\prime}: H^{i} M(X) \rightarrow Z^{i} M(X) \subset M^{i}(X) \rightarrow Q^{i} M(X),
$$

(DGLA) $\quad h: H_{i-1} \mathcal{L}(X) \rightarrow Z_{i-1} \mathcal{L}(X) \subset \mathcal{L}_{i-1}(X) \rightarrow Q \mathcal{L}_{i-1}(X) \cong s^{-1} \tilde{H}_{i}(X)$

where the first map in each sequence is a chosen splitting and the last is projective onto indecomposables. The identifications $H_{i-1} \mathcal{L}(X) \cong \pi_{i}(X), Q^{i} M(X) \cong$ $\operatorname{Hom}\left(\pi_{i} X, \mathbf{Q}\right)$ complete the description. An alternative approach is given by observing that

$$
M(X) \rightarrow Q M(X) \quad \text { and } \quad \mathcal{L}(X) \rightarrow Q \mathcal{L}(X)
$$

are (co)chain maps. Hence, there are induced maps

$$
H^{*} M(X) \rightarrow H^{*} Q M(X) \cong Q M(X), \quad H \mathcal{L}(X) \rightarrow H Q \mathcal{L}(X) \cong Q \mathcal{L}(X) .
$$

The existence of the indicated isomorphisms is equivalent to the minimality of $M(X)$ and $\mathcal{L}(X)$ respectively. We will use the following notation:

$$
R(X)=\operatorname{Image}\left(h^{\prime}\right), \quad S(X)=\operatorname{Image}(h) .
$$

Finally, we recall the dual notions of operators and cooperators (see $[\mathbf{H i}]$ ). If $F \rightarrow E \rightarrow B$ is a fibration, then the homotopy lifting property provides an operation of $\Omega B$ on $F, c: F \times \Omega B \rightarrow F$. For $\alpha \in \pi_{n} F, \mu \in \pi_{n+1} B \cong \pi_{n}(\Omega B), c_{\#}(\alpha, \mu)=$ $\alpha+\partial_{\#} \mu$ where $\partial_{\#}$ is the connecting homomorphism. The induced map of minimal models $C: M(F) \rightarrow M(F) \otimes M(\Omega B)$ induces a map of indecomposables $Q C: Q F \rightarrow$ $Q F \oplus Q(\Omega B)$ which takes the form $Q C(x)=\left(x, \partial^{\#} x\right)$, where $\partial^{\#}$ is the dual of $\partial_{\#}$.

To a cofibration $X \rightarrow Y \rightarrow C$ there is associated a cooperation $c: C \rightarrow C \vee \Sigma X$. The induced map on homology is given by $c_{*}(x)=\left(x, \partial_{*} x\right)$, where $\partial_{*}$ is the connecting homomorphism. This is, of course, $Q C$, the map induced on indecomposables by the DGLA map $C: \mathcal{L}(C) \rightarrow \mathcal{L}(C) \bigsqcup \mathcal{L}(\Sigma X)$ corresponding to c. The symbol "Џ" denotes the free Lie product. (It is not hard to show that $\mathcal{L}(C \vee \Sigma X) \cong \mathcal{L}(C) \amalg \mathcal{L}(\Sigma X)$.)

3. Statements, proofs and corollaries. In this section, we first state the minimal model version of Theorem 1 and prove some corollaries of it. The dual situation is then considered. Finally, minimal model proofs of the main decomposition results are given.

THEOREM $1^{\prime}$. If $F \stackrel{i}{\rightarrow} E \stackrel{p}{\rightarrow} B$ is a fibre sequence, then the DGA model of $F$ has a decomposition

$$
M(F) \cong\left(L\left(V^{\prime}\right), 0\right) \otimes\left(L\left(V^{\prime \prime}\right), d\right)
$$

where $V^{\prime}=R(F) / R(F) \cap \operatorname{Im} Q i$. Consequently,

$$
H^{*}(F) \cong L\left(V^{\prime}\right) \otimes H^{*}\left(L\left(V^{\prime \prime}\right), d\right) .
$$
$Q F$.

REMARK. Recall that $Q i$ denotes the induced map on indecomposables $Q E \rightarrow$

COROLLARY a. If $\operatorname{dim}\left(R^{2 n}(F) / R^{2 n}(F) \cap \operatorname{Im} Q i\right) \geq 1$, then $H^{2 k n}(F) \neq 0$ for all $k \geq 1$.

ProOF. The hypothesis ensures that there exists a nontrivial $x \in V^{\prime}$ of even degree. Hence, $x$ is a polynomial generator of $L\left(V^{\prime}\right)$, so $x^{k} \neq 0$ for all $k \geq 1$. By Theorem $\mathbf{1}^{\prime}$ we are done. Q.E.D. 
COROLlaRY b. If $H^{2}(E) \rightarrow H^{2}(F)$ is not surjective, then $H^{2 k}(F) \neq 0$ for all $k \geq 1$.

PROOF. If $X$ is 1-connected, then the inductive construction of the minimal model $M(X)$ shows that $Q^{1} X=0$ and $Q^{2} X=H^{2}(X)$. Thus, $R^{2}(F)=Q^{2} F$ and the hypothesis is equivalent to the nonsurjectivity of $Q i: Q^{2} E \rightarrow Q^{2} F$. By Corollary a we are done. Q.E.D.

REMARK. Corollary b is proven in [GLSW] by entirely different methods and is listed as Theorem 2 in that work. Furthermore, results of this nature were obtained by Gottlieb in his study of the evaluation map. In particular, Corollary 3 and Theorems 4 and 5 of [G2] yield an alternative proof of Corollary b.

THEOREM $2^{\prime}$. If $X \stackrel{f}{\rightarrow} Y \stackrel{j}{\rightarrow} C$ is a cofibre sequence, then the DGLA model of $C$ has a decomposition,

$$
\mathcal{L}(C) \cong\left(F\left[V^{\prime}\right], 0\right) \coprod\left(F\left[V^{\prime \prime}\right], d\right)
$$

where $V^{\prime}=S(C) / S(C) \cap \operatorname{Im} j_{*}$. Consequently,

$$
\pi_{*}(\Omega C) \cong F\left[V^{\prime}\right] \coprod\left(F\left[V^{\prime \prime}\right], d\right) .
$$

REMARK. Note the duality between Theorems $1^{\prime}$ and $2^{\prime}$. Specifically, note that the tensor product $\otimes$ and the free Lie product $\amalg$ are the coproducts in the categories DGA and DGLA respectively.

The next two results exemplify the heuristic nature of Eckmann-Hilton duality. Although they are formally similar to Corollaries a and b, the dimension restriction of Corollary a does not dualize because the attributes of free graded Lie algebras differ significantly from those of free graded commutative algebras.

COROLlaRY c. If $\operatorname{dim}\left(S_{n}(C) / S_{n}(C) \cap \operatorname{Im} j_{*}\right) \geq 2$, then $\pi_{k(n-1)+1}(C) \neq 0$ for all $k \geq 1$.

ProOF. The hypothesis ensures that the free graded Lie algebra $F\left[V^{\prime}\right]$ has at least two generators. Consequently, there exists a nontrivial basic product in the degrees indicated (see $[\mathbf{W}])$. Q.E.D.

Corollary d. If $\operatorname{dim}\left(\operatorname{Coker}\left(j_{*}\right)_{3}\right) \geq 2$, then $\pi_{2 k+1}(C) \neq 0$ for all $k \geq 1$.

PROOF. Because the differential of $\mathcal{L}(C)$ is decomposable, the indecomposable space $Q_{2} \mathcal{L}(C) \cong s^{-1} \tilde{H}_{3}(C)$ consists of cycles. Hence $S_{3}(C)=s^{-1} \tilde{H}_{3}(C)$ and Corollary c then applies. Q.E.D.

For the proofs of Theorems $1^{\prime}$ and $2^{\prime}$ we recall two facts which were used in $\S 1$. If $X=\Omega Y$, then Hopf's Theorem implies that $X$ has the homotopy type of a product of Eilenberg-Mac Lane spaces. Hence, the differential $d_{X}$ is trivial and $M(X) \cong H^{*}(X) \cong L\left(\operatorname{Hom}\left(\pi_{*} X, \mathbf{Q}\right)\right)$. If $X=\Sigma Y$, then $X$ has the homotopy type of a wedge of spheres. Hence, the DGLA differential $d_{X}$ is trivial and $\mathcal{L}(X) \cong$ $\pi_{*}(\Omega X) \cong F\left[s^{-1} \tilde{H}_{*}(X)\right]$ by the Bott-Samelson Theorem.

PROOF OF THEOREM $1^{\prime}$. From the definition of $V^{\prime}$, we see that the restriction of $\partial^{\#}: Q F \rightarrow Q(\Omega B)$ to $V^{\prime}$ is injective. Take a graded vector space splitting of $\left.\partial^{\#}\right|_{V^{\prime}}$ and extend it freely to a DGA map

$$
\Delta: M(\Omega B)=(L(Q \Omega B), 0) \rightarrow\left(L\left(V^{\prime}\right), 0\right) .
$$


Here, we have used the isomorphism, $Q \Omega B \cong \operatorname{Hom}\left(\pi_{*}(\Omega B), \mathbf{Q}\right)$ together with the remarks preceding the proof. Those remarks also allow us to represent the DGA $\left(L\left(V^{\prime}\right), 0\right)$ as a product of Eilenberg-Mac Lane spaces $K$. The inclusion $\left(L\left(V^{\prime}\right), 0\right) \rightarrow$ $M(F)$ then is represented by a map $\alpha: F \rightarrow K$. Let $\mathcal{F}$ denote the homotopy fibre of $\alpha$ and let $\beta: M(F) \rightarrow M(\mathcal{F})$ denote the induced map.

Note that the definition of $V^{\prime}$ implies that $\alpha_{\#}: \pi_{*}(F) \rightarrow \pi_{*}(K) \cong$ $\operatorname{Hom}\left(V^{\prime}, \mathbf{Q}\right)$ is surjective. By the exactness of the homotopy sequence of the fibration $\mathcal{F} \rightarrow F \rightarrow K$ we then have direct sum decompositions,

$$
\pi_{*}(F) \cong \pi_{*}(\mathcal{F}) \oplus \pi_{*}(K), \quad Q F \cong Q \mathcal{F} \oplus V^{\prime} .
$$

Now, the composition $(\beta \otimes \Delta) C: M(F) \rightarrow M(F) \otimes M(\Omega B) \rightarrow M(\mathcal{F}) \otimes\left(L\left(V^{\prime}\right), 0\right)$ induces the map on indecomposables,

$$
(Q \beta \oplus Q \Delta) Q C: Q F \rightarrow Q \exists \oplus V^{\prime} .
$$

The decomposition of $Q F$ given above, the description of $Q C$ given in $\S 2$ and the definitions of $Q \beta, Q \Delta$ clearly imply that $(Q \beta \oplus Q \Delta) Q C$ is an isomorphism. Because minimal DGA's are freely generated by their indecomposables, we then obtain the desired isomorphism,

$$
M(F) \cong\left(L\left(V^{\prime}\right), 0\right) \otimes M(\mathcal{F}) . \quad \text { Q.E.D. }
$$

Since the proof of Theorem $2^{\prime}$ is dual to that of Theorem $1^{\prime}$, we suppress some of the details.

ProOF OF ThEOREM $2^{\prime}$. A splitting of the injection $V^{\prime} \rightarrow H_{*}(\Sigma X)$ induces $\mathcal{L}(\Sigma X) \rightarrow\left(F\left[V^{\prime}\right], 0\right)$ using the fact that $d_{\Sigma X}=0$. Let $S$ denote a wedge of spheres which represents $\left(F\left[V^{\prime}\right], 0\right)$ and let $S \rightarrow C$ represent the inclusion $\left(F\left[V^{\prime}\right], 0\right) \rightarrow$ $\mathcal{L}(C)$. Denote the homotopy cofibre of $S \rightarrow C$ by $C$. Because $V^{\prime} \rightarrow H_{*}(C)$ is injective, the exactness of the homology sequence of $S \rightarrow C \rightarrow C$ gives a graded vector space decomposition,

$$
H_{*}(C) \cong H_{*}(C) \oplus V^{\prime} .
$$

Let $\mathcal{L}(C) \rightarrow \mathcal{L}(C)$ model $C \rightarrow C$ and consider the composition,

$$
\mathcal{L}(C) \stackrel{C}{\rightarrow} \mathcal{L}(C) \coprod \mathcal{L}(\Sigma X) \rightarrow \mathcal{L}(C) \coprod\left(F\left[V^{\prime}\right], 0\right) .
$$

Again, the induced map on indecomposables (i.e., $\left.s^{-1} \tilde{H}_{*}()\right)$ is easily seen to be an isomorphism. Since minimal DGLA's are freely generated by their indecomposables, we obtain the isomorphism

$$
\mathcal{L}(C) \cong\left(F\left[V^{\prime}\right], 0\right) \coprod \mathcal{L}(C) \text {. Q.E.D. }
$$

ACKNOWLEDGEMENTS. I would like to thank Dan Gottlieb, Reinhard Schultz and Joe Neisendorfer for several conversations in connection with this paper. I would also like to express my appreciation to the referee for his suggestions, questions and comments. In particular, the formulation of Theorem $1^{\prime}$ is his.

4. Appendix. The proof of Theorem $1^{\prime}$ shows that the restriction of $\partial^{*}$ to $L\left(V^{\prime}\right)$ is injective. Subsequently, Felix and Thomas have observed that, indeed, the following holds. 
COROLlaRY e. $L\left(V^{\prime}\right)=\operatorname{Im}\left(\partial^{*}: H^{*}(F) \rightarrow H^{*}(\Omega B)\right)$.

The proof of this result derives from the work of Felix and Thomas on the operation of rational holonomy (which in one form is given by)

$$
H_{*}(\Omega B) \otimes H^{*}(F) \rightarrow H^{*}(F) .
$$

The reader is referred to their paper, Sur l'operation d'holonomie rationelle, which is to appear in the Lecture Notes in Mathematics, Stockholm, 1983, for details. See especially $§ 3.2$.

Corollary e follows from the fact that $\partial^{*}$ is a map of $H_{*}(\Omega B)$-modules, so $\operatorname{Im} \partial^{*}$ is an $H_{*}(\Omega B)$-submodule of $H^{*}(\Omega B)$. The module structure here derives from the path fibration of $B$. The following Lemma, together with Theorem $1^{\prime}$, complete the proof.

LEMMA. Every subalgebra $M$ of $H^{*}(\Omega B)$ which is an $H_{*}(\Omega B)$-module is of the form $L(W)$, where $W$ is a vector space of indecomposables of $H^{*}(\Omega B)$.

PROOF (FELIX-PRIVATE COMMUNICATION). For convenience we write $M(B)$ $=(L(X), d)$ and $H^{*}(\Omega B)=M(\Omega B)=(L(\bar{X}), 0)$, where $\bar{X}$ is $X$ shifted down one degree.

Let $\alpha \in M \subset L(\bar{X})$ and write

$$
\alpha=\sum_{i \in I} a_{i} \bar{x}(i, 1) \cdots \bar{x}\left(i, p_{i}\right), \quad a_{i} \in \mathbf{Q}, p=\inf \left(p_{i}\right) .
$$

By duality, we may choose $u(i, j) \in \operatorname{Prim}\left(H_{*}(\Omega B)\right) \cong \pi_{*}(\Omega B)$ with

$$
\langle x(i, j), \mathrm{s} u(i, j)\rangle=1
$$

where $\mathrm{s}=$ suspension by one degree and $\mathrm{s} \bar{x}=x$.

Now, each $u(i, j)$ acts as a derivation so that the element of $H_{*}(\Omega B)$, $\left(u(i, 1) \cdots \hat{u}(i, j) \cdots u\left(i, p_{i}\right)\right)=u$ acts as (see Felix-Thomas),

$$
\begin{aligned}
u \cdot \alpha & =\left(u(i, 1) \cdots \hat{u}(i, j) \cdots u\left(i, p_{i-1}\right)\right) \cdot\left(u\left(i, p_{i}\right) \cdot \alpha\right) \\
& =\tilde{a}_{i} \bar{x}(i, j)+\mu(i, j)
\end{aligned}
$$

where $\tilde{a}_{i} \in \mathbf{Q}$ and $\mu(i, j) \in L(\bar{X})^{+} \cdot L(\bar{X})^{+}$. (For example,

$$
\begin{aligned}
u_{1} u_{2} \cdot\left(\bar{x}_{1} \bar{x}_{2} \bar{x}_{3}\right) & =u_{1}\left(0 \pm \bar{x}_{1} \cdot 1 \cdot \bar{x}_{3}+0\right)=u_{1}\left( \pm \bar{x}_{1} \bar{x}_{3}\right) \\
& \left.= \pm 1 \cdot \bar{x}_{3}+0= \pm \bar{x}_{3} .\right)
\end{aligned}
$$

But $u \cdot \alpha \in M$, so $\bar{x}(i, j)-v(i, j) \in M$ where $v(i, j)=-\left(1 / \tilde{a}_{i}\right) \mu(i, j)$.

Let $\bar{y}(i, j)=\bar{x}(i, j)-v(i, j)$ be a new set of generators for $M(\Omega B)$. Then replace $\bar{x}(i, j)$ in the expression for $\alpha$ by $\bar{y}(i, j)+v(i, j)$. We obtain $\alpha=\tilde{\alpha}+\beta$ where $\tilde{\alpha}$ is a sum of products of the indecomposables $\{\bar{y}\}$ and $\beta$ is a sum of words of length $\geq p+1$.

We may continue this process inductively until the lengths of words in $\beta$ exceed degree of $\alpha$. Then we must have $\beta=0$. Hence, $\alpha$ is obtained as a sum of products of indecomposables of $M(\Omega B)$. Q.E.D. 
Felix and Thomas apply Corollary e to prove the interesting result (Theorem 4.6 in their work),

THEOREM FT. If $F \rightarrow E \rightarrow B$ is a fibration of 1-connected spaces and $H^{*}(F)$ is a finitely generated $H_{*}(\Omega B)$-module, then the following are equivalent:

1. $H^{*}(F)$ is nilpotent (i.e., there exists an $n>0$ such that $\left(H^{+}\right)^{n}=0$ ).

2. The composition $h \partial_{\#}: \pi_{n+1}(B) \rightarrow H_{n}(F)$ is trivial above a certain dimension and for each even $n$.

I would like to thank Yves Felix for explaining Corollary e to me as well as bringing to my attention the beautiful application of Theorem 1', Theorem FT.

\section{REFERENCES}

[BL] H. J. Baues and J. M. Lemaire, Minimal models in homotopy theory, Math. Ann. 225 (1977), 219-242.

[C] H. Cartan, La transgression dans un groupe de Lie et dans un espace fibré principal, Colloque de Topologie, Bruxelles, 1950, pp. 57-71.

[FT] Y. Felix and J. C. Thomas, Homotopie rationelle, dualité et complémentarite des modèles, Bull. Soc. Math. Belg. Ser. A 33 (1981), 7-19.

[G1] D. H. Gottlieb, Evaluation subgroups of homotopy groups, Amer. J. Math. 91 (1969), 729-756.

[G2] _ Witnesses, transgressions, and the evaluation map, Indiana Univ. Math. J. 24 (1975), 825-836.

[GLSW] M. Gotay, et al., Closed forms on symplectic fibre bundles, Comment. Math. Helv. 58 (1983), 617-621.

[GM] P. Griffiths and J. Morgan, Rational homotopy theory and differential forms, Birkhäuser, Boston, Mass., 1981.

[H] H. B. Haslam, $G$-spaces $\bmod F$ and $H$-spaces $\bmod F$, Duke Math. J. 38 (1971), 671-679.

[H1] S. Halperin, Rational fibrations, minimal models, and fibrings of homogeneous spaces, Trans. Amer. Math. Soc. 244 (1978), 199-224.

[H2] _ Lectures on minimal models, Publ. IRMA Lille 3 (1981).

[H3] __ Finiteness in the minimal models of Sullivan, Trans. Amer. Math. Soc. 230 (1977), 173-199.

[Hi] P. Hilton, Homotopy theory and duality, Gordon and Breach, New York, 1965.

[HT] S. Halperin and J. C. Thomas, Rational equivalence of fibrations with fibre $G / K$, Canad. J. Math. 34 (1982), 31-43.

[N] J. Neisendorfer, Lie algebras, coalgebras and rational homotopy theory for nilpotent spaces, Pacific J. Math. 74 (1978), 429-460.

[Q] D. Quillen, Rational homotopy theory, Ann. of Math. (2) 90 (1969), 205-295.

[T] D. Tanre, Homotopie rationnelle: modèles de Chen, Quillen, Sullivan, Lecture Notes in Math., vol. 1025, Springer-Verlag, 1983.

[W] G. Whitehead, Elements of homotopy theory, Graduate Texts in Math., no. 61, SpringerVerlag, 1978.

Department of Mathematics, Purdue University, West lafayette, Indiana 47907 44115

Current address: Department of Mathematics, Cleveland State University, Cleveland, Ohio 